\title{
Pixel Value Ordering Based Reversible Data Hiding with Novel MPBS Strategy
}

\author{
M. Mahasree, N. Puviarasan, P. Aruna
}

\begin{abstract}
Recently, Reversible Data Hiding (RDH) techniques has gained much attention in many sensitive fields such as remote sensing, archive management, military and medical image processing systems. This is due to the lossless data extraction ability of RDH schemes. The primary goal of $R D H$ schemes is to achieve high embedding rates while maintaining the quality of cover objects. For achieving better performance, Pixel Value Ordering (PVO) based reversible data hiding schemes have been proposed. PVO refers to the process of ranking the pixels in blocks and then modifying the pixels according to some embedding rules/conditions. So far, the existing PVO techniques have considered neighborhood pixels at unit distance. In this paper, an improved RDH using block based PVO scheme is proposed which exploits the pixel correlation efficiently by increasing the block size and applying a novel Median Pixel based Block Selection Strategy (MPBS). When block size is increased, the ordering of pixels is changed after embedding. So, to extract the secret data in a lossless manner, the secret bits are swapped in accordance with their corresponding Stego pixels' index order. Also, the overflow and underflow conditions are effectively handled using Location Map. Experimental results show the better performance of the proposed RDH technique with the existing technique.
\end{abstract}

Keywords: Reversible data hiding, pixel value ordering, pixel correlation, block selection, index order, location map.

\section{INTRODUCTION}

Information hiding is one of the research areas, which has grown quickly in the recent years. In this technology world, protecting secret from intruders continues to be one of the top challenges in the field of information technology and telecommunication system. Data hiding provides transmission from one place to another in a secured manner. According to reversibility, the data hiding schemes are classified into two types: They are i) Distortion-free or Lossless or Reversible Data Hiding and ii) Fragile or Irreversible Data Hiding. Reversible Data Hiding (RDH) methods are those that allow embedding data inside a cover

Revised Manuscript Received on February 15, 2020.

* Correspondence Author

M. Mahasree*, Department of Computer Science and Engineering, Annamalai University, Chidambaram, India. Email: mahasree05@gmail.com

N. Puviarasan, Department of Computer and Information Science, Annamalai University, Chidambaram, India. Email: npuvi2410@yahoo.in

P. Aruna, Department of Computer Science and Engineering, Annamalai University, Chidambaram, India. Email: arunapuvi@yahoo.co.in

(C) The Authors. Published by Blue Eyes Intelligence Engineering and Sciences Publication (BEIESP). This is an open access article under the CC BY-NC-ND license (http://creativecommons.org/licenses/by-nc-nd/4.0/) media and later not only the hidden data can be retrieved but the exact cover media can also be recovered [1,2]. One of the main application areas of RDH include medical database management where sensitive patient details are to be protected [3]. In the irreversible methods it is not possible to recover the original media exactly, after the hidden data is retrieved. The goal of $\mathrm{RDH}$ is to increase the stego quality and assuring the reversibility after embedding. There are three types under which reversible hiding is carried on. They are Difference Expansion (DE), Histogram Shifting (HS) and Prediction-Error Expansion (PEE) based RDH schemes [4]. In $\mathrm{DE}$, the correlation between two neighbor pixels is used for embedding secret bits. This method achieved good imperceptibility but low embedding rates. Thus, Histogram based RDH schemes were proposed to overcome this limitation. HS based schemes work on the histogram of the cover media [5]. The peak and empty bins are utilized to embed secret bits. PEE is the extension of DE where the target pixel is predicted by context pixels from its neighbor and then the prediction error is used for expansion embedding [6]. Other than these techniques, PVO is the recently developed RDH technique where block wise embedding is done based on largest/smallest pixel in the block. This technique gives promising results than other existing methods.

The rest of paper is organized as follows. Section II briefly describes the related works. Section III presents the proposed Block PVO method by improving the block size and block selection. Experimental results are provided in Section IV. Finally, Section V concludes this paper.

\section{LITERATURE SURVEY}

Wenguang et al. proposed a novel pixel-based pixel value grouping (PPVG) in which embedding is done based on the difference between maximum and minimum context pixels. Priority is given to pixels present in smooth regions. [7]. Bo Ou et al. discussed the PVO embedding in two-dimensional (2D) space. They divide the carrier into different blocks and utilized error pairs for data embedding. 2D mapping is used to get back the original cover as well as secret data [8]. Wenguang et al. extended the original PVO into a general form namely k-pass PVO in which Type- 1 and Type-2 embedding capacity are increased [9]. Biswapati Jana proposed a high payload reversible data hiding scheme using a weighted matrix using interpolation technique [10].

Pascal Maniriho and Tohari Ahmad presented an improved reversible data hiding scheme based on difference expansion and modulus function. [11].

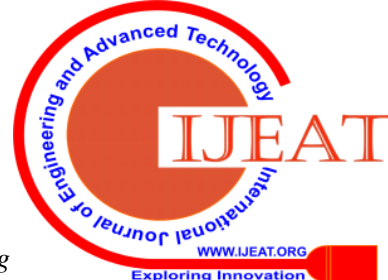

$\&$ Sciences Publication 
Weng et al. proposed a reversible hiding scheme using flexible image block division and adaptive pixel modifications. Both the highest and lowest intensity valued pixels are modified to embed more bits [12].

Siren Cai and Xinlu Gui proposed a data hiding scheme based on reference pixel and histogram shifting [13]. Jung proposed a scheme that considers three pixels block with pixel sorting and prediction. They divided the cover image into non-overlapping blocks in ascending order. The maximum and minimum prediction errors are calculated to check whether the data could be embedded or not. This method gives better stego quality with reasonable embedding capacity. However, the pixels correlation is not effectively utilized [14]. Liu and Lee proposed an increased capacity based reversible steganography based on the relative performance in row or column of the cover image [15]. Hence, with improved pixels correlation, PVO based RDH is proposed to increase the embedding capacity.

\section{PROPOSED WORK} procedures of the proposed MPBS_RDH system.

Let $C$ and $C$ ' be the carrier and stego image. The cover image is the carrier of the secret data. After embedding, the cover image is termed as stego image. The size of cover and stego image is denoted as $M \mathrm{x} N$. Let $S$ denote the binary array of secret bits. The block index where the last bit is embedded $(k x, k y)$, the threshold value $(t)$, size of the compressed location map $\left(l_{c l m}\right)$ and their values (CLM) information are the additional parameters required for the retrieval process. These parameters are finally embedded into the stego image using the same procedure as that of embedding secret bits. Fig. 1 shows the overall block diagram of the proposed RDH technique.

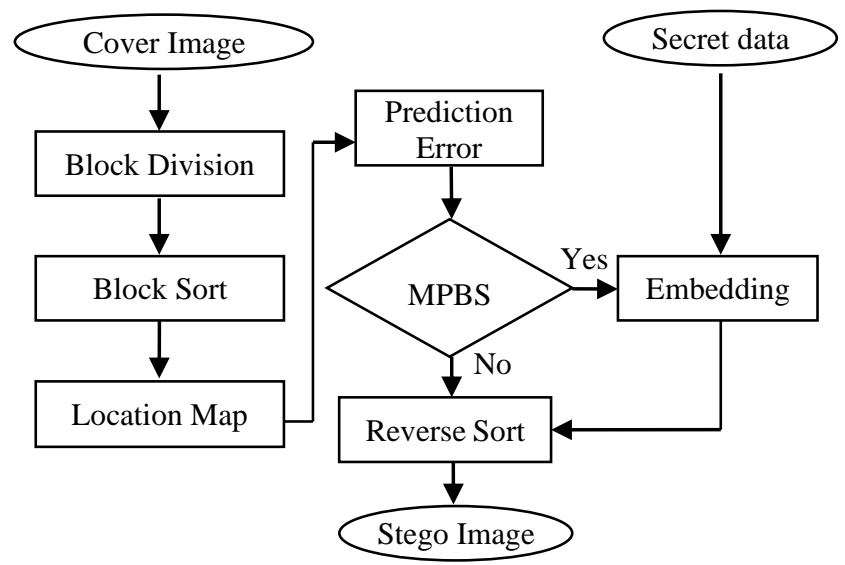

Fig. 1. The block diagram of proposed MPBS_RDH technique

\section{A. Embedding Procedure}

When secret data is to be embedded, the cover image undergoes a pre-processing stage of Block Division. The Proposed PVO technique begins by dividing the cover image into $5 \times 5$ blocks in a horizontal manner. Based on the size of cover image, block division is carried out adaptively (i.e., the cover size is taken as multiples of 5 and the remaining rows/columns are kept unchanged). Raster scan ordering is preferred in this block division process. Each block is
This section presents the embedding and extraction

processed as five separate 1x5 sub-blocks. In each sub-block, the pixels are sorted in ascending order and their indices are kept in a matrix $B$, to ensure re-ordering after the embedding process. The median pixels in all sub-blocks are the reference pixels to be used at extraction stage and so kept unchanged. The absolute differences among the median values are used for sub-block selection. The embedding of secret bits is done by applying embedding rule. While embedding, there arise two cases. One is Overflow where the maximum pixel value 255 is modified into 256. Other case is Underflow where the minimum pixel value 0 is changed to -1 . To solve this problem, a Location Map ( $L M$ ) is used before embedding. Initially, $L M$ is set as an empty array. When ' 1 ' or ' 254 ' is encountered, then binary value ' 0 ' is inserted into the $L M$. When ' 0 ' or ' 255 ' is encountered, then binary value ' 1 ' is inserted into the $L M$ and the values are changed to ' 1 ' or ' 254 ' respectively. Thus, the length of location map is image-dependant. Then the location map size is compressed by applying lossless arithmetic compression (CLM). The details of the embedding and extraction algorithms are given below.

\section{Algorithm 1: Proposed embedding method \\ Input: Cover image $C$, Secret bit array $S$ of length $l$ \\ Output: Stego image $D$ \\ Step 1: Get the input image $C$ of size $512 \times 512$ \\ Step 2: Get the secret data $S$ which is in binary form \\ Step 3: Divide $C$ into $5 \times 5$ non-overlapping blocks \\ Step 4: For each block, sort all the five $1 \mathrm{x} 5$ sub-blocks in ascending order

$$
\left[\left(r_{i}, r_{i+1}, r_{i+2}, r_{i+3}, r_{i+4}\right), B\right]=\operatorname{sort}\left(q_{i}, q_{i+1}, q_{i+2}, q_{i+3}, q_{i+4}\right)
$$

Step 5: For all sub-blocks, calculate the difference values $\left(d_{0}, d_{1}, d_{3}, d_{4}\right)$ on each side of the median.

$$
\begin{gathered}
d_{0}=r_{i}-r_{i+2} ; d_{1}=r_{i+1}-r_{i+2} \\
d_{3}=r_{i+3}-r_{i+2} ; d_{4}=r_{i+4}-r_{i+2}
\end{gathered}
$$

Step 6: Calculate the noise level (NL) of the block as the sum of absolute differences between every two consecutive median pixels of its 1x5 sub-blocks.

$$
N L=\sum\left|d_{\text {median }}\right|
$$

Step 7: Compute threshold value $t(0 \leq t \leq 255)$ which determines the complexity of a sub-block.

Step 8: If the median of a sub-block is less than or equal to threshold $t$ (median $\leq t$ ), that sub-block is selected for embedding. Otherwise, it is kept unchanged.

Step 9: If the sub-block is selected, then using the Embedding rule, embed the secret bit $s$ from Secret array $S$ into the difference values as follows:

$$
\begin{aligned}
& d_{0}^{\prime}=\left\{\begin{array}{cl}
0, & \text { if } d_{0}=0 \\
d_{0}-s, & \text { if } d_{0}=-1 \\
d_{0}-1, & \text { if } d_{0}<-1
\end{array}\right. \\
& d_{3}^{\prime}=\left\{\begin{array}{cl}
0, & \text { if } d_{3}=0 \\
d_{3}+s, & \text { if } d_{3}=1 \\
d_{3}+1, & \text { if } d_{3}>1
\end{array}\right.
\end{aligned}
$$

where s denotes the secret bit 0 or 1 . Similarly, the secret bits are embedded into $d_{1}^{\prime}$ and $d_{4}^{\prime}$ using eq.3 and eq.4 respectively. 
Step 10: Calculate the new pixel pairs as follows:

$$
\begin{aligned}
& r_{i}^{\prime}=r_{i+2}+d_{0}^{\prime} ; \quad r_{i+1}^{\prime}=r_{i+2}+d_{1}^{\prime} \\
& r_{i+3}^{\prime}=r_{i+2}+d_{3}^{\prime} ; r_{i+4}^{\prime}=r_{i+2}+d_{4}^{\prime}(5)
\end{aligned}
$$

Step 11: The embedded stego pixels are obtained by re-arranging the new pairs back to their original positions.

$$
\left(q_{i}^{\prime}, q_{i+1}^{\prime}, q_{i+2}^{\prime}, q_{i+3}^{\prime}, q_{i+4}^{\prime}\right)=\operatorname{rsort}\left[\left(r_{i}^{\prime}, r_{i+1}^{\prime}, r_{i+2}^{\prime}, r_{i+3}^{\prime}, r_{i+4}^{\prime}\right), B\right]
$$

Step 12: Thus, the stego image $D$ is obtained.

Now, the additional information has to be included in the stego image. So, least significant bits (LSB) in first 44 pixels of stego image are saved as $S_{l s b}$. Then replace these LSB by the additional information ( $k x-9$ bits, $k y-9$ bits, $t-8$ bits, $l_{c l m}-18$ bits). Finally, embed $s_{l s b}+C L M$ pixels into the remaining stego pixels.

\section{B. Extraction Procedure}

At the receiver end, the stego image is processed same as the embedding steps in a reverse manner. Firstly, the additional information is extracted from the stego image. Then, the secret data are extracted with the help of additional information. The detailed algorithm for extraction is described as follows.

Algorithm 2: Proposed extraction method

Input: Stego image $D$

Output: Secret data, Cover image

Step 1: Get the input Stego $D$ of size $512 \times 512$

Step 2: Extract all the required additional information

Step 3: Divide $D$ into $5 \times 5$ non-overlapping blocks

Step 4: For each block, sort all the five $1 \times 5$ sub-blocks of stego pixels in ascending order

$$
\left[\left(r_{i}^{\prime}, r_{i+1}^{\prime}, r_{i+2}^{\prime}, r_{i+3}^{\prime}, r_{i+4}^{\prime}\right), B\right]=\operatorname{sort}\left(q_{i}^{\prime}, q_{i+1}^{\prime}, q_{i+2}^{\prime}, q_{i+3}^{\prime}, q_{i+4}^{\prime}\right)
$$

Step 5: Calculate the difference values $\left(d_{0}^{\prime}, d_{1}^{\prime}, d_{3}^{\prime}, d_{4}^{\prime}\right)$ on each side of the Median

$$
\begin{gathered}
d_{0}^{\prime}=r_{i}^{\prime}-r_{i+2}^{\prime} ; d_{1}^{\prime}=r_{i+1}^{\prime}-r_{i+2}^{\prime} \\
d_{3}^{\prime}=r_{i+3}^{\prime}-r_{i+2}^{\prime} ; d_{4}^{\prime}=r_{i+4}^{\prime}-r_{i+2}^{\prime}
\end{gathered}
$$

Step 6: By checking the threshold $t$, extract the secret bit $s$ as follows:

$$
\begin{gathered}
d_{0}=\left\{\begin{array}{cc}
0, & \text { if } d_{0}^{\prime}=0 \\
d_{0}^{\prime} \text { and } s=0, & \text { if } d_{0}^{\prime}=-1 \\
d_{0}^{\prime}+1 \text { and } s=1, & \text { if } d_{0}^{\prime}=-2 \\
d_{0}^{\prime}+1, & \text { if } d_{0}^{\prime}<-2
\end{array}\right. \\
d_{3}=\left\{\begin{array}{cc}
0, & \text { if } d_{3}^{\prime}=0 \\
d_{3}^{\prime} \text { and } s=0, & \text { if } d_{3}^{\prime}=1 \\
d_{3}^{\prime}-1 \text { and } s=1, & \text { if } d_{3}^{\prime}=2 \\
d_{3}^{\prime}-1, & \text { if } d_{3}^{\prime}>2
\end{array}\right.
\end{gathered}
$$

Similarly, the secret bits are extracted from $d_{1}$ and $d_{4}$ using eq. 7 and eq. 8 respectively.

Step 7: Calculate the original cover pixel pairs as follows:

$$
\begin{gathered}
r_{i}=r_{i+2}^{\prime}+d_{0} ; r_{i+1}=r_{i+2}^{\prime}+d_{1} \\
r_{i+3}=r_{i+2}^{\prime}+d_{3} ; r_{i+4}=r_{i+2}^{\prime}+d_{4} ; r_{i+2}=r_{i+2}^{\prime}
\end{gathered}
$$

Step 8: If $\left(r_{i}, r_{i+1}\right)$ have same values after secret bit extraction, then based on their corresponding positions in stego image, the extracted secret bits have to be changed as:

$$
\operatorname{swap}\left(S_{d 0}, S_{d 1}\right) \quad \text {,if } B\left(a_{i}\right)>B\left(a_{i+1}\right)
$$

$$
\operatorname{swap}\left(S_{d 3}, S_{d 4}\right) \text {,if } B\left(a_{i+3}\right)>B\left(a_{i+4}\right)
$$

where $B$ denotes the index positions of $a_{n}$ in $D$ and $S_{d n}$ denotes the extracted secret bits.

Step 9: The original cover pixels are obtained by re-arranging the new pixel pairs $\left(r_{i}, r_{i+1}, r_{i+2}, r_{i+3}, r_{i+4}\right)$ back to their original positions.

$$
\left(q_{i}, q_{i+1}, q_{i+2}, q_{i+3}, q_{i+4}\right)=\operatorname{rsort}\left[\left(r_{i}, r_{i+1}, r_{i+2}, r_{i+3}, r_{i+4}\right), B\right]
$$

Step 10: The original cover and secret data are recovered.

After the extraction of all secret data, the unmodified image is obtained by reversing the overflow pixels and underflow pixels using the location map.

\section{Illustration For The Proposed RDH Method}

An example of the proposed embedding technique is shown in Fig.2. To illustrate the embedding, a $1 \times 5$ sub-block with value $\left(q_{i}, q_{i+1}, q_{i+2}, q_{i+3}, q_{i+4}\right)=(175,174,174,176,178)$ is taken from lena image. After sorting, the sorted pixels are $\left(r_{i}, r_{i+1}, r_{i+2}, r_{i+3}, r_{i+4}\right)=(174,174,175,176,178)$. The difference values are calculated as $d_{0}=-1, d_{1}=-1, d_{3}=1, d_{4}=3$. Let the secret bits be $s=(\mathbf{0}, \mathbf{1}, 0)$. According to embedding rule, the difference values with one are embedded as follows: $d_{0}=-1, d_{1}=-2, d_{3}=1, d_{4}=4$. The new embedded pixels are calculated as $\left(r_{i}^{\prime}, r_{i+1}^{\prime}, r_{i+2}, r_{i+3}^{\prime}, r_{i+4}^{\prime}\right)=(174,173,175,176,179)$. The centre pixel is kept unchanged so that it is used as a reference pixel at the receiver side. It is observed that due to embedding, the sorting order is changed here (173 comes after 174). This will be solved during extraction process. The pixels are put back to their original positions $\left(q_{i}^{\prime}, q_{i+1}^{\prime}, q_{i+2}^{\prime}, q_{i+3}^{\prime}, q_{i+4}^{\prime}\right)=(175,174,173,176,179)$.

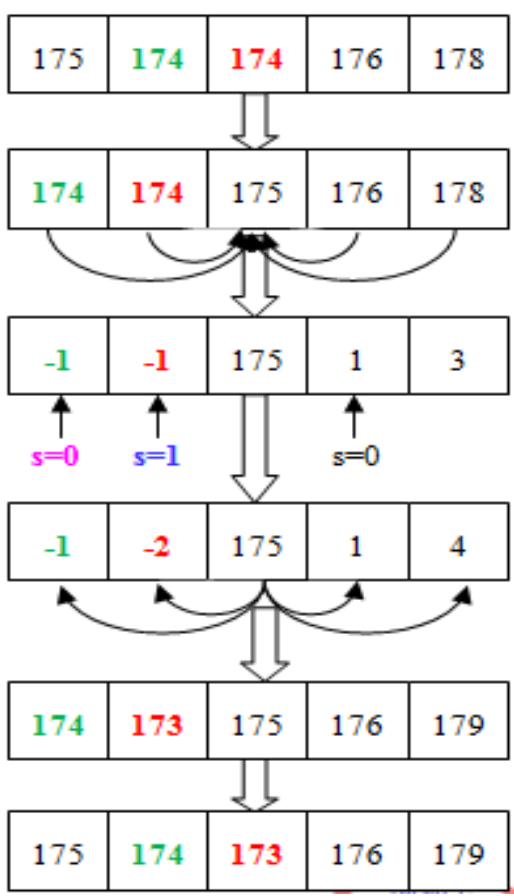

Fig. 2.Embedding phase of the proposed MPBS_RDH

Published By:

Retrieval Number: B4248129219/2020@BEIESP DOI: 10.35940/ijeat.B4248.029320 Journal Website: www.ijeat.org

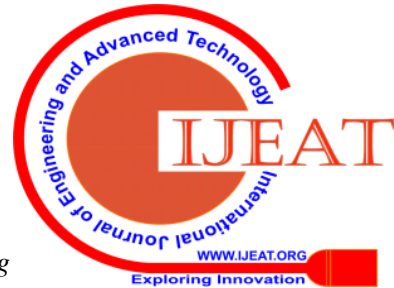


Fig.3 shows the extraction method. Now, at extraction process the stego pixels $(175,174,173,176,179)$ are sorted in ascending

order as $\left(r_{i}^{\prime}, r_{i+1}^{\prime}, r_{i+2}^{\prime}, r_{i+3}^{\prime}, r_{i+4}^{\prime}\right)=(173,174,175,176,179)$. The difference values between pixels and median generated are $d_{0}^{\prime}=-2, d_{1}^{\prime}=-1, d_{3}^{\prime}=1, d_{4}^{\prime}=4$. According to extraction rules, $\left(r_{i}, r_{i+1}, r_{i+2}, r_{i+3}, r_{i+4}\right)=(174,174,175,176,178)$ are the recovered pixels and the secret bits are $s=(\mathbf{1}, \mathbf{0}, 0)$. The first and second pixels are similar $\left(r_{i}=r_{i+1}\right)$. So, check for their order of indices. The first pixel $\left(r_{i}^{\prime}=173\right)$ is at $3^{\text {rd }}$ position. The second pixel $\left(r_{i+1}^{\prime}=174\right)$ is at $2^{\text {nd }}$ position. Hence, swap their secret bits as $s=(\mathbf{0}, \mathbf{1}, 0)$. Finally, the original pixels are recovered by applying reverse sorting procedure, $\left(q_{i}, q_{i+1}, q_{i+2}, q_{i+3}, q_{i+4}\right)=(175,174,174,176,178)$

\begin{tabular}{|c|c|c|c|c|}
\hline 175 & 174 & 173 & 176 & 179 \\
\hline 173 & 174 & 175 & 176 & 179 \\
\hline-2 & -1 & 175 & 1 & 4 \\
\hline$\stackrel{\nabla}{s=1}$ & $\underset{s=0}{\searrow}$ & & $\stackrel{\nabla}{\mathrm{v}=0}$ & \\
\hline-1 & -1 & 175 & 1 & 3 \\
\hline 174 & 174 & 175 & 176 & 178 \\
\hline 174 & 174 & 175 & 176 & 178 \\
\hline$s=0$ & $s=1$ & & $\stackrel{\nabla}{\mathrm{v}}=0$ & \\
\hline 175 & 174 & 174 & 176 & 178 \\
\hline
\end{tabular}

Fig. 3. Extraction phase of the proposed MPBS_RDH

\section{EXPERIMENTAL RESULTS}

Proposed method is applied to standard grayscale and color images for experimentation. The implementation was done using Python. The cover images are of size $512 \times 512$. The results are compared with existing PVO based Reversible data hiding techniques. The performance measures used to evaluate the proposed system are Peak-Signal-to-Noise Ratio, Embedding Capacity and Structural Similarity Index Measure.

\section{A. Performance Metrics}

The image quality of the embedded image can be evaluated using PSNR as follows:

$$
P S N R=10 * \log \left(\frac{255^{2}}{M S E}\right)
$$

$$
M S E=\frac{1}{M N} \sum_{i=0}^{M} \sum_{j=0}^{N}(P(i, j)-Q(i, j))^{2}
$$

where PSNR is Peak Signal-to-Noise Ratio and MSE is Mean Square Error between original image $P(i, j)$ and reference image $\mathrm{Q}(i, j)$ with dimension $M \times N$. Structural Similarity Index Measure is given by,

$$
\begin{gathered}
\operatorname{SSIM}(A, B)=\frac{\left(2 \mu_{x} \mu_{y}+c_{1}\right)\left(2 \sigma_{x y}+c_{2}\right)}{\left(\mu_{x}^{2}+\mu_{y}^{2}+c_{1}\right)\left(\sigma_{x}^{2}+\sigma_{y}^{2}+c_{2}\right)} \\
c_{1}=\left(k_{1} L\right)^{2}, \quad c_{2}=\left(k_{2} L\right)^{2}
\end{gathered}
$$

where $\mu_{\mathrm{x}}$ and $\mu_{\mathrm{y}}$ are average values; $\sigma_{\mathrm{x}}{ }^{2}$ and $\sigma_{\mathrm{y}}{ }^{2}$ denotes variance, $\sigma_{\mathrm{xy}}$ is the covariance of $\mathrm{x}$ and $\mathrm{y}$; $\mathrm{c}_{1}$ and $\mathrm{c}_{2}$ are variables for stabilization, $\mathrm{L}=255$ denotes pixel range, $\mathrm{k}_{1}=0.01, \mathrm{k}_{2}=0.03$ are default constants and $\mathrm{x}, \mathrm{y}$ are original and reference images respectively. Embedding capacity denotes the total number of bits that can be embedded into the cover image.

\section{B. Performance Analysis for Grayscale Images}

Fig.4 shows the standard gray-scale images used for experiments.

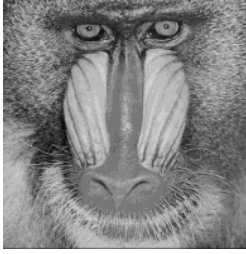

$$
\text { Baboon }
$$

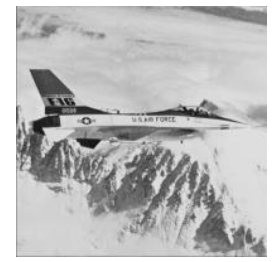

Jetplane

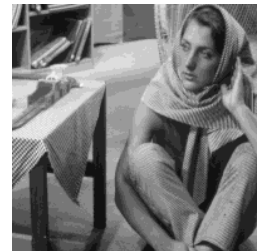

Barbara

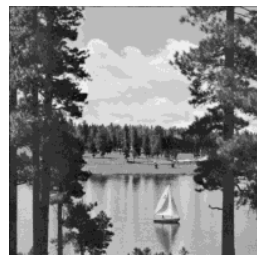

Lake

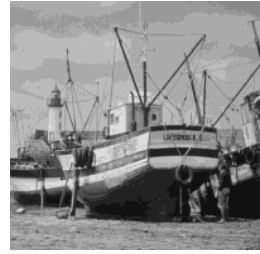

Boat

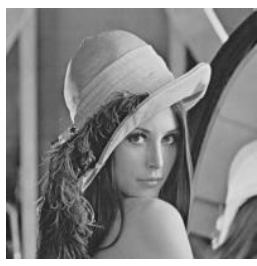

Lena
Fig. 4. Grayscale test images (SIPI database)

Table-I shows the performance of the proposed MPBS based RDH technique with the existing techniques for test images under different metrics. In Jung's method, the block size is $3 \times 1$ and the embedding locations are single pixels at both sides of the centre pixel. So, the capacity is low when compared to our proposed method which uses $1 \times 5$ blocks with two embedding locations on both sides of the centre pixel. For example, the lena image shows highest increase in capacity from 34228 bits to 45480 bits for the proposed RDH.

The PSNR comparison shows that there is not much difference between the existing methods and the proposed method. This slight variation in PSNR of our proposed method is due to the increase in total number of bits embedded i.e., the capacity and PSNR are inversely proportional to each other. The SSIM tells that pixels have strong dependencies among them especially when they are closer to each other. From the table, it is proved that the structural similarity between cover and stego images is higher for our proposed MPBS_RDH method. 
Table- I: Performance of the proposed method in terms of Capacity, PSNR and SSIM for grayscale images

\begin{tabular}{|l|c|c|c|c|c|c|c|c|c|}
\hline \multirow{2}{*}{$\begin{array}{c}\text { Grayscale } \\
\text { Images }\end{array}$} & \multicolumn{3}{|c|}{ Jung [12] } & \multicolumn{3}{c|}{ Liu and Lee [13] } & \multicolumn{4}{c|}{ Proposed System } \\
\cline { 2 - 11 } & $\begin{array}{c}\text { Capacity } \\
\text { (bits) }\end{array}$ & $\begin{array}{c}\text { PSNR } \\
\text { (dB) }\end{array}$ & SSIM & $\begin{array}{c}\text { Capacity } \\
\text { (bits) }\end{array}$ & $\begin{array}{c}\text { PSNR } \\
\text { (dB) }\end{array}$ & SSIM & $\begin{array}{c}\text { Capacity } \\
\text { (bits) }\end{array}$ & $\begin{array}{c}\text { PSNR } \\
\text { (dB) }\end{array}$ & SSIM \\
\hline Baboon & 14241 & 50.32 & 0.9968 & 14100 & 50.27 & 0.9959 & 14453 & 50.29 & 0.9982 \\
\hline Barbara & 27263 & 50.77 & 0.9855 & 32690 & 50.79 & 0.9875 & 33840 & 50.02 & 0.9956 \\
\hline Boat & 37445 & 51.17 & 0.9779 & 39657 & 51.03 & 0.9775 & 43536 & 50.35 & 0.9964 \\
\hline Jetplane & 46551 & 51.75 & 0.9693 & 45259 & 51.58 & 0.9702 & 52501 & 50.77 & 0.9964 \\
\hline Lake & 26957 & 50.76 & 0.9882 & 26986 & 50.67 & 0.9877 & 30249 & 49.90 & 0.9965 \\
\hline Lena & 34228 & 51.05 & 0.9793 & 38736 & 51.05 & 0.9842 & 45480 & 50.42 & 0.9944 \\
\hline
\end{tabular}
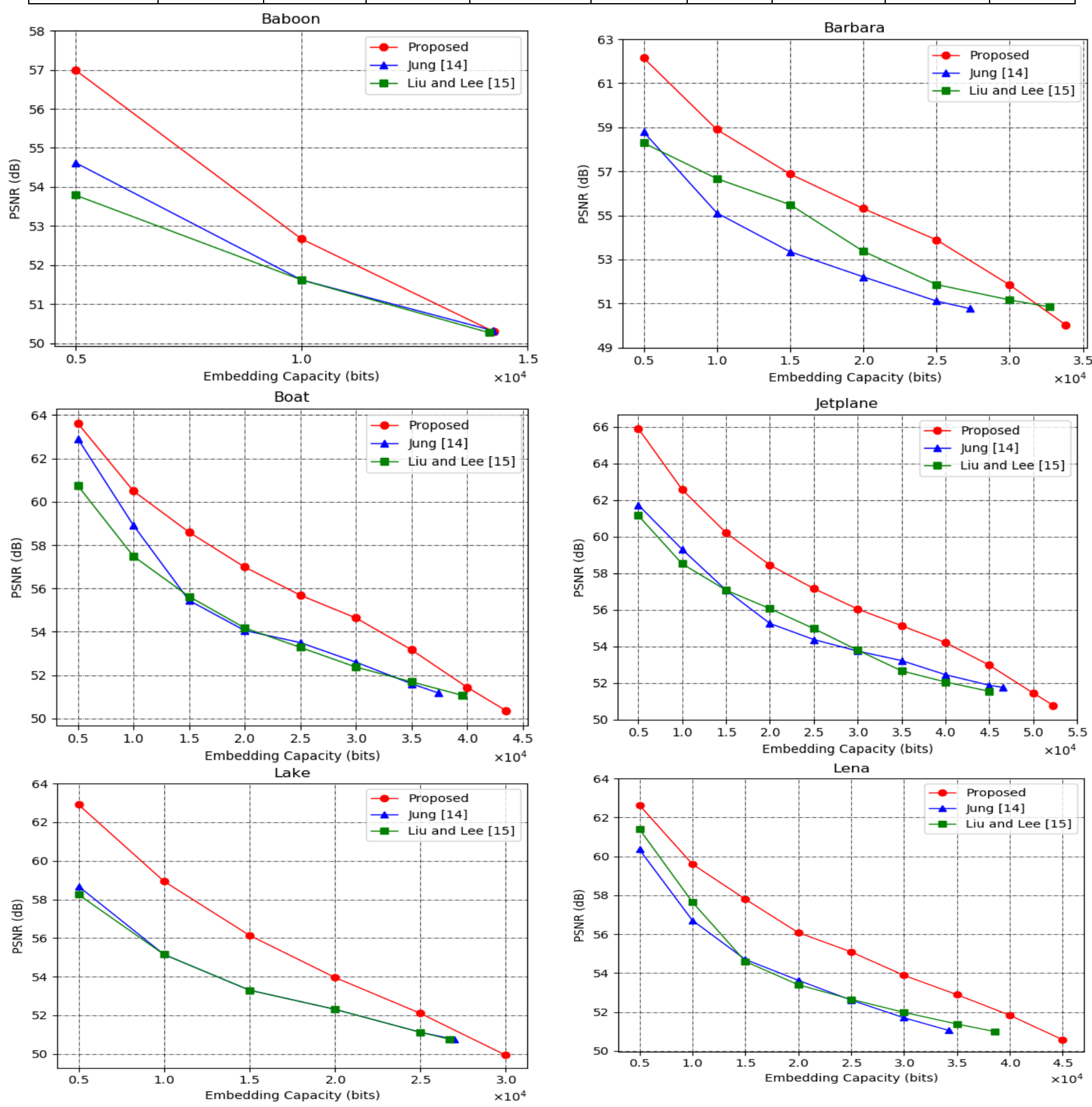

Fig. 5.Rate-Distortion comparison of proposed method with other methods

Rate-distortion curves of proposed MPBS_RDH with respect to test image quality are shown in Fig.5. The embedding capacity is varied from five thousand bits to its maximum with a step size of 5000 bits. The figure also shows that the capacity of our RDH algorithm is superior to that of existing methods, while maintaining a high PSNR, 
demonstrating the superiority of the proposed MPBS_RDH hiding scheme.

\section{Performance Analysis for Color Images}

Fig. 5 shows the grayscale images used for experiments.

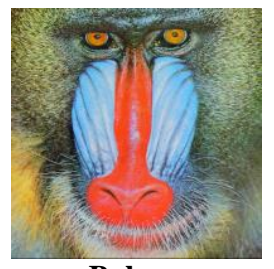

Baboon

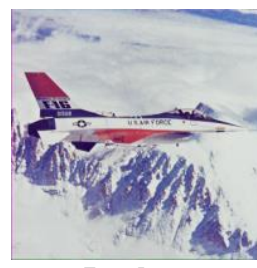

Jetplane

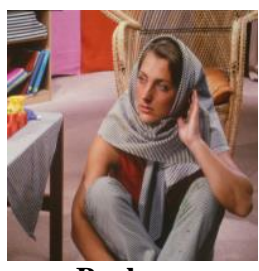

Barbara

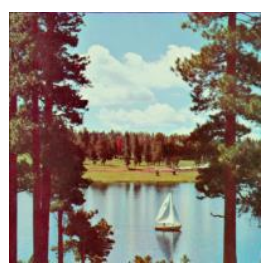

Lake

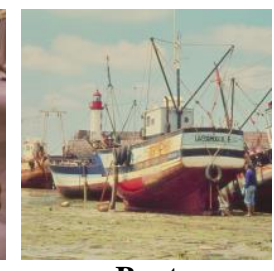

Boat

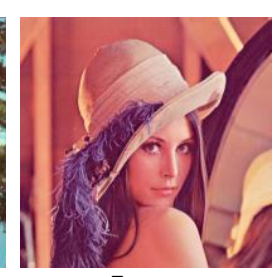

Lena
Fig. 6.Color test images

Table-II shows the performance of the proposed technique in terms of PSNR and SSIM for color images with a fixed capacity of 10000 . This table shows how the capacity is partitioned in three planes R, G and B when optimal threshold is provided.

Table- II: Performance of the proposed method in terms of Capacity, PSNR and SSIM for Color images

\begin{tabular}{|c|c|c|c|c|c|}
\hline $\begin{array}{l}\text { Color } \\
\text { Images }\end{array}$ & $\begin{array}{c}\text { Capacity } \\
\text { (bits) }\end{array}$ & \begin{tabular}{|c|} 
Optimal \\
Threshold \\
(t)
\end{tabular} & $\begin{array}{c}\text { R, G, B } \\
\text { Partition } \\
\text { (bits) }\end{array}$ & $\begin{array}{c}\text { PSNR } \\
\text { (dB) }\end{array}$ & SSIM \\
\hline \multirow{3}{*}{ Baboon } & \multirow{3}{*}{10000} & \multirow{3}{*}{9} & 4598 & \multirow{3}{*}{60.82} & \multirow{3}{*}{0.9996} \\
\hline & & & 520 & & \\
\hline & & & 3882 & & \\
\hline \multirow{3}{*}{ Barbara } & \multirow{3}{*}{10000} & \multirow{3}{*}{4} & 3013 & \multirow{3}{*}{63.13} & \multirow{3}{*}{0.9996} \\
\hline & & & 4964 & & \\
\hline & & & 2023 & & \\
\hline \multirow{3}{*}{ Boat } & \multirow{3}{*}{10000} & \multirow{3}{*}{2} & 2752 & \multirow{3}{*}{65.97} & \multirow{3}{*}{0.9997} \\
\hline & & & 7248 & & \\
\hline & & & 0 & & \\
\hline \multirow{3}{*}{ Jetplane } & \multirow{3}{*}{10000} & \multirow{3}{*}{1} & 3716 & \multirow{3}{*}{66.30} & \multirow{3}{*}{0.9998} \\
\hline & & & 4466 & & \\
\hline & & & 1818 & & \\
\hline \multirow{3}{*}{ Lake } & \multirow{3}{*}{10000} & \multirow{3}{*}{3} & 1591 & \multirow{3}{*}{61.19} & \multirow{3}{*}{0.9996} \\
\hline & & & 3004 & & \\
\hline & & & 5405 & & \\
\hline \multirow{3}{*}{ Lena } & \multirow{3}{*}{10000} & \multirow{3}{*}{4} & 10000 & \multirow{3}{*}{64.71} & \multirow{3}{*}{0.9997} \\
\hline & & & 0 & & \\
\hline & & & 0 & & \\
\hline
\end{tabular}

Table-III gives the performance of proposed technique in terms of PSNR and SSIM for color images. This table shows the maximum achievable capacity of the proposed technique in various color images. It also separately displays the capacity partition of R, G and B channels.
Table- III: Maximum Capacity obtained for proposed method

\begin{tabular}{|c|c|c|c|c|}
\hline Images & $\begin{array}{c}\text { Total } \\
\text { Capacity } \\
\text { (bits) }\end{array}$ & $\begin{array}{l}\text { Maximum } \\
\text { Capacity for } \\
\text { R, G, B } \\
\text { (bits) }\end{array}$ & $\begin{array}{c}\text { PSNR } \\
\text { (dB) }\end{array}$ & SSIM \\
\hline \multirow{3}{*}{ Baboon } & \multirow{3}{*}{42846} & 15966 & \multirow{3}{*}{49.19} & \multirow{3}{*}{0.9985} \\
\hline & & 12780 & & \\
\hline & & 14100 & & \\
\hline \multirow{3}{*}{ Barbara } & \multirow{3}{*}{87731} & 28235 & \multirow{3}{*}{49.93} & \multirow{3}{*}{0.9971} \\
\hline & & 32794 & & \\
\hline & & 26702 & & \\
\hline \multirow{3}{*}{ Boat } & \multirow{3}{*}{137019} & 45676 & \multirow{3}{*}{50.47} & \multirow{3}{*}{0.9966} \\
\hline & & 48092 & & \\
\hline & & 43251 & & \\
\hline \multirow{3}{*}{ Jetplane } & \multirow{3}{*}{150865} & 49169 & \multirow{3}{*}{50.52} & \multirow{3}{*}{0.9958} \\
\hline & & 49701 & & \\
\hline & & 51995 & & \\
\hline \multirow{3}{*}{ Lake } & \multirow{3}{*}{80693} & 24822 & \multirow{3}{*}{49.20} & \multirow{3}{*}{0.9959} \\
\hline & & 23867 & & \\
\hline & & 32004 & & \\
\hline \multirow{3}{*}{ Lena } & \multirow{3}{*}{112227} & 47471 & \multirow{3}{*}{50.18} & \multirow{3}{*}{0.9961} \\
\hline & & 34652 & & \\
\hline & & 30104 & & \\
\hline
\end{tabular}

\section{CONCLUSION}

In this paper, a new improved block based reversible data hiding method has been proposed. The proposed method could embed a maximum of about 4 bits of the secret data in each $1 \times 5$ sub-block. The pixels are arranged in increasing intensity order and the difference values are calculated. Based on these values, the threshold value for a given capacity decides whether a secret bit could be hidden or not The experimental results showed the performance of the proposed system with respect to embedding capacity was better than previous method and quality of stego images were also improved. It is also applied to color images for proving the efficiency of our proposed MPBS strategy. Experimental results showed that the proposed MPBS_RDH method outperforms the existing methods.

\section{REFERENCES}

1. Yun Q. Shi, "Reversible Data Hiding", Lecture Notes in Computer Science, Springer, vol. 3304, pp. 1-12, 2005.

2. Revathi Nath H A and Jeena R S, "An Efficient Algorithm for Reversible Data Hiding in Encrypted Images by RRBE", International Journal of Engineering and Advanced Technology (IJEAT), vol. 6, pp.74-78, 2016

3. Shubhangi Kolhe et al., "Image Encryption Using Reversible Data Hiding by Reserving Room before Encryption", International Journal of Engineering and Advanced Technology (IJEAT), vol. 4, pp.53-55, 2015

4. Chin-Feng Lee and Hsing-Ling Chen, "Adjustable prediction-based reversible data hiding”, Digital Signal Processing, Elsevier, vol. 22, pp. 941-953, 2012.

5. Tai WL et al., "Reversible data hiding based on histogram modification of pixel differences", IEEE Transactions on Circuits and Systems for Video Technology, vol. 19, 2009. 
6. Hong et al., "Reversible data hiding for high quality images using modification of prediction errors", The Journal of Systems and Software, vol. 82, pp.1833-1842, 2009.

7. Wenguang et al., "Improved reversible data hiding using pixel-based pixel value grouping", Optik - International Journal for Light and Electron Optics, Elsevier, pp. 1-15, 2017.

8. Bo $\mathrm{Ou}$ et al., "High-fidelity reversible data hiding based on pixel-value-ordering and pairwise prediction-error expansion”, Journal of Visual Communication and Image Representation, Elsevier, pp. 12-23, 2016.

9. Wenguang et al., "Reversible data hiding using multi-pass pixel value ordering and prediction error expansion", Journal of Visual Communication and Image Representation, Elsevier, 2017.

10. Biswapati Jana, "High payload reversible data hiding scheme using weighted matrix", Optik, Elsevier, vol. 127, pp. 3347-3358, 2016.

11. Maniriho, P. and Ahmad, T., "Information hiding scheme for digital images using difference expansion and modulus function”, Journal of King Saud University - Computer and Information Sciences, Elsevier, pp. 1-13, 2018.

12. Weng et al., "Reversible data hiding based on flexible block-partition and adaptive block-modification strategy", Journal of Visual Communication and Image Representation, Elsevier, pp. 1-33, 2016.

13. Siren Cai and Xinlu Gui, "An Efficient Reversible Data Hiding Scheme Based on Reference Pixel and Block Selection", International Conference on Intelligent Information Hiding and Multimedia Signal Processing, IEEE, 2013.

14. Ki-Hyun Jung, "A high-capacity reversible data hiding scheme based on sorting and prediction in digital images", Multimedia Tools and Applications, Springer, vol. 76, pp. 13127-13137, 2016.

15. Hsing-Han Liu and Chuan-Min Lee, "High-capacity reversible image steganography based on pixel value ordering", EURASIP Journal on Image and Video Processing, Springer, vol. 54, pp. 1-15, 2019.

\section{AUTHORS PROFILE}

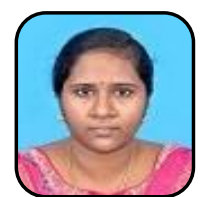

M. Mahasree received her M.E degree in Computer Science and Engineering from Annamalai University in 2017. She is currently doing Ph.D in Computer Science and Engineering, Annamalai University. Her research areas include Digital Image Processing and Data Security.

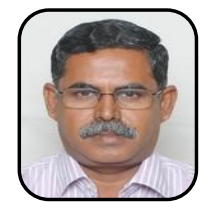

N. Puviarasan completed Ph.D in Computer Science and Engineering from Annamalai University in 2016. He has 32 years of teaching experience. He has published 45 papers in international journals, 14 papers in international conferences and published 6 Book chapters. He is currently working as a Professor in Department of Computer and Information Science, Faculty of Science, Annamalai University.

P. Aruna received her M.Tech Degree from IIT Delhi. She completed Ph.D in Computer Science and Engineering from Annamalai University in 2006. She has 29 years of teaching experience. She has produced 11 PhDs in Computer Science and Engineering. She is currently guiding 3 research scholars in the Department of Computer Science and Engineering. She has published 85 papers in international journals, 29 papers in international conferences and published 8 Book chapters. She is currently working as the Head of the Department in Department of Computer Science and Engineering, Faculty of Engineering and Technology, Annamalai University. 